\title{
XMCD Analysis Beyond Standard Procedures
}

\author{
H. Wende ${ }^{1, *}$, A. Scherz ${ }^{2, *}$, C. Sorg ${ }^{3, *}$, K. Baberschke*, E.K.U. Gross ${ }^{*}$, H. Appel, \\ K. Burke ${ }^{\dagger}$, J. Minár**, H. Ebert**, A.L. Ankudinov* and J.J. Rehr* \\ ${ }^{*}$ Fachbereich Physik, Freie Universität Berlin, Arnimallee 14, D-14195 Berlin-Dahlem, Germany. \\ ${ }^{\dagger}$ Department of Chemistry and Chemical Biology, Rutgers University, 610 Taylor Rd, Piscataway, NJ 08854 \\ ** Institut für Physikalische Chemie, Universität München, Butenandtstraße 5-13, D-81377 München, Germany \\ Department of Physics, University of Washington, Seattle, Washington 98195-1560, USA
}

\begin{abstract}
We show that the standard analysis procedures as sum rule application and multipole-moment analysis for XMCD spectra can fail for magnetic samples of present interest. Two examples will be given: 1) The induced magnetic moments in ultrathin films of the light $3 d$ elements $\mathrm{Ti}, \mathrm{V}$ and $\mathrm{Cr}$ at the interface to $\mathrm{Fe}$ cannot be determined by the XMCD sum rule or multipole-moment analysis at the $L_{2,3}$ edges. This is due to correlation effects which result in the deviation of the intensity ratio (branching ratio) from its statistical value. To address this point we established a double-pole approximation within timedependent density functional theory. 2) The analysis of the $L_{2,3} \mathrm{XMCD}$ of rare earth elements is not only hampered by the appearance of electric quadrupolar contributions (E2) in addition to the dipolar contributions (E1). Even after separation of the two, one determines the wrong sign of the induced $5 d$ moment by the sum rules. This originates from the spin dependence of the transition matrix elements. To tackle these difficulties we compare the experimental spectra to $a b$ initio calculations of the entire isotropic XAS and the dichroic signal.
\end{abstract}

Keywords: XMCD, failure sum rule and multipole moment analysis, induced magnetism, light $3 d$ metals and rare earth metals PACS: 78.70.Dm, 78.70.Ls, 31.15.Ew, 31.70.Hq, 71.20.Be

\section{INTRODUCTION}

About three decades after theoretical prediction of the $\mathrm{x}$ ray magnetic circular dichroism (XMCD) [1] and about two decades after the experimental realization [2] this technique (XMCD) has been demonstrated to be a unique tool for the element-specific analysis of magnetic properties. By means of the so-called sum rules [3, 4] and multipole-moment analysis [5] the spin and orbital moments can be determined by analyzing the integrals of the dichroic spectra and their spectral shape, respectively. These methods became standard procedures for the analysis of XMCD data and are nowadays used by a large number scientists. However, various assumptions are made in the derivation of these analysis procedures as pointed out in the original works on the sum rule analysis and other recent works (see e.g. $[6,7,8,9]$ ). As we will demonstrate in the present work this has the consequence that the standard analysis of the $L_{2,3}$ edges XMCD fails for magnetic systems of present interest as e.g. induced moments in light $3 d$ elements as well as the $5 d$ magnetism in rare earth metals.

\footnotetext{
${ }^{1}$ New address: Angewandte Physik - AG Wende, Fachbereich Physik, Universität Duisburg-Essen, Lotharstr. 1, D-47048 Duisburg, Germany

${ }^{2}$ New address: SSRL, Stanford Linear Accelerator Center, 2575 Sand Hill Road, Menlo Park, California 94025, USA

${ }^{3}$ New address: Max-Planck-Institut für Metallforschung, Heisenbergstraße 3, D-70569 Stuttgart, Germany
}

\section{INDUCED MAGNETISM IN LIGHT $3 d$ METALS}

Light $3 d$ metals like Ti and $\mathrm{V}$ exhibit induced magnetic moments at the interface to the $3 d$ ferromagnets like Fe. For a detailed study of these effects we studied prototype trilayer systems as shown in Fig. 1 (a). The questions are if indeed one determines induced moments in $\mathrm{V}$ and what is the size and the orientation of the induced spin and orbital moments as indicated by the arrows. For
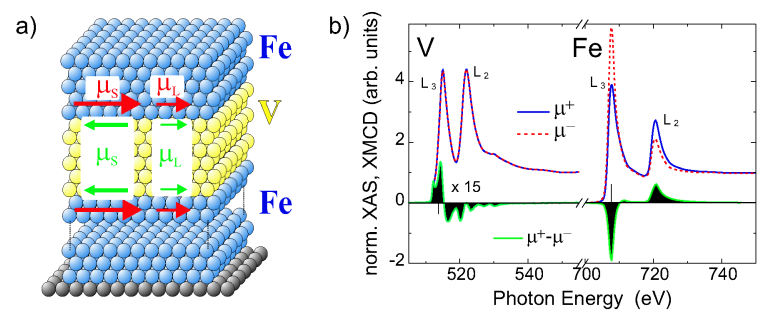

FIGURE 1. (a) Schematic representation of a prototype $\mathrm{Fe} / \mathrm{V} / \mathrm{Fe}(110)$ trilayer. (b) Normalized XAS for right $\left(\mu^{+}\right)$and left $\left(\mu^{-}\right)$circularly polarized $\mathrm{x}$-rays (top) and the corresponding XMCD at the $\mathrm{V}$ and $\mathrm{Fe} L_{2,3}$ edges of a $\mathrm{Fe} / \mathrm{V}_{4} / \mathrm{Fe}$ trilayer [10]. The V XMCD was multiplied by a factor 15 for a clearer presentation.

these systems the advantage of the element specificity of the XMCD is obvious as revealed in Fig. 1(b): The x-ray absorption coefficients $\mu^{+}$and $\mu^{-}$for right and left circularly polarized $\mathrm{x}$-rays and the corresponding XMCD at the $\mathrm{V}$ and $\mathrm{Fe} L_{2,3}$ edges of an $\mathrm{Fe} / \mathrm{V}_{4} / \mathrm{Fe}$ trilayer with 4

CP882, X-ray Absorption Fine Structure-XAFS13 edited by B. Hedman and P. Pianetta

(C) 2007 American Institute of Physics 978-0-7354-0384-0/07/\$23.00 

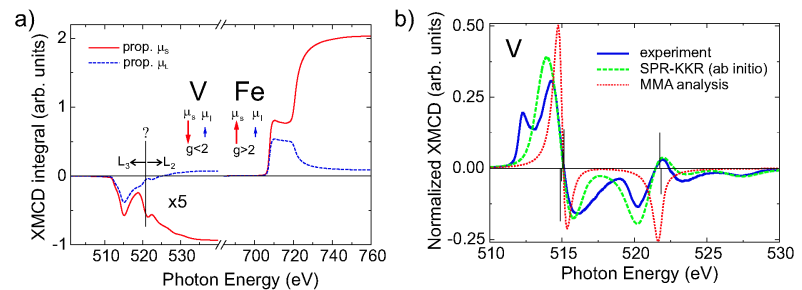

FIGURE 2. a) Experimental XMCD integrals at the $\mathrm{V}$ and Fe $L_{2,3}$ edges for a $\mathrm{Fe}_{0.9} \mathrm{~V}_{0.1}$ alloy [7] which are used for the standard XMCD sum rule analysis. b) Result of the multipolemoment analysis (MMA) (dotted line) of the experimental XMCD data (solid line). The MMA results are compared to ab initio calculations (dashed line) [11].

ML V thickness are presented [10]. A clear XMCD signal from the Fe buffer and a much smaller signal originating from the induced $\mathrm{V}$ moment can be seen. The positive XMCD signal at the $\mathrm{V} L_{3}$ edge in comparison to the negative signal at the $\mathrm{Fe} L_{3}$ edge (see arrows in Fig. 1(b)) shows that the induced moment is aligned antiparallel to Fe. However, when applying the standard analysis procedures to determine the size of the induced spin and orbital moments in $\mathrm{V}$ it turns out that these procedures fail. The results of the sum rule analysis and the multipolemoment analysis are shown in Fig. 2 for a $\mathrm{Fe}_{0.9} \mathrm{~V}_{0.1}$ alloy [11]. First, we apply the integral sum rule analysis depicted in Fig. 2(a). The analysis at the $\mathrm{Fe} L_{2,3}$ edges provides spin and orbital moments in agreement to theoretical SPR-KKR calculations [11]. Furthermore, the Fe total moment for this alloy is in agreement with a polarized neutron study (PNS) [12]. However, the results of the sum rule analysis at the $\mathrm{V} L_{2,3}$ edges completely disagree with the experimental neutron investigation and the theoretical SPR-KKR calculation. It turns out that the spin moment and also the total moment as determined from the sum rules are about a factor 5 to small compared to theory $\left(\mu_{S}(\right.$ sum rule $)=-0.20 \mu_{B}$ in contrast to $\mu_{S}$ (SPR$\left.\mathrm{KKR})=-1.01 \mu_{B}\right)$ and the PNS measurements $[11,7]$. Since the integral sum rule analysis ignores the spectral shape of the XMCD one could argue that it would be more appropriate to fit the detailed fine structure in the $\mathrm{V}$ XMCD with the multipole-moment analysis (MMA) [5]. To test this we compared the MMA results to ab initio SPR-KKR calculations by using the various parameters entering into the MMA procedure from the SPR-KKR calculation [11]. Hence, we compare theoretical results among each other. The results are presented in Fig. 2(b). First of all the $a b$ initio calculation reproduces mainly all the fine structures of the experimental XMCD spectra. Interestingly, the MMA procedures resembles the asymmetry at the $L_{3}$ edge. However, the procedure fails to reproduce the asymmetry at the $L_{2}$ edge completely. This demonstrates that the two standard analysis procedures

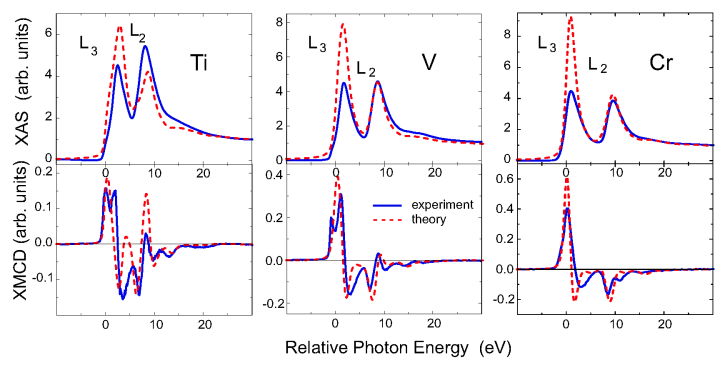

FIGURE 3. Normalized XAS (above) and XMCD spectra (below) for the light $3 d \mathrm{TM} \mathrm{Ti}, \mathrm{V}$, and $\mathrm{Cr}$ at the $L_{2,3}$ edges: (solid lines) experimental data versus (dashed lines) $a b$ initio calculations [14].

fail for this light $3 d$ element. To overcome this difficulty we used the results for the $\mathrm{Fe}_{0.9} \mathrm{~V}_{0.1}$ alloy as an experimental standard by adopting the SPR-KKR results for $\mu_{S}$ and $\mu_{L}$. The absolute moments for the trilayer systems are then determined by scaling to this standard $[11,7]$. This is possible since the spectral shape of the V XMCD of the trilayers is similar to the one of the $\mathrm{FeV}$ alloy.

For a more detailed understanding of the failure of the standard analysis procedures we performed a systematic investigation along the $3 d$ series [13]. The results for the the light $3 d$ elements Ti, $\mathrm{V}$ and $\mathrm{Cr}$ are presented in Fig. 3. The same trilayer setup as depicted in Fig. 1(a) was analyzed. Hence, induced moments in Ti and V are probed, whereas uncompensated moments for the antiferromagnet $\mathrm{Cr}$ are measured. The detailed analysis reveals that the lighter the $3 d$ element is, the larger becomes the deviation of the sum rule results from the theoretical predictions $[14,7]$. The question arises if this trend can be related to a systematic change in the spectra along the $3 d$ series? Such a relation can be determined when analyzing the so-called branching ratio. This ratio is defined as $B=A_{3 / 2} /\left(A_{3 / 2}+A_{1 / 2}\right)$ where $A_{j}$ is the area under isotropic XAS of the $j^{\text {th }}$ subshell, i.e. the $L_{3}$ and $L_{2}$ white line intensities. The statistical branching ratio is calculated from the $j=3 / 2$ and $j=1 / 2$ manifolds and yields

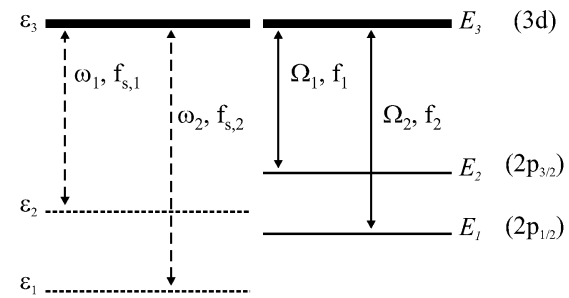

FIGURE 4. Schematic illustration of the DPA model. The model describes the shifts of the excitation energies (uncorrelated $\omega_{i}$ and correlated $\Omega_{i}$ ) and the changes in corresponding oscillator strengths $f_{i}$ in the presence of an excited core hole [15]. 


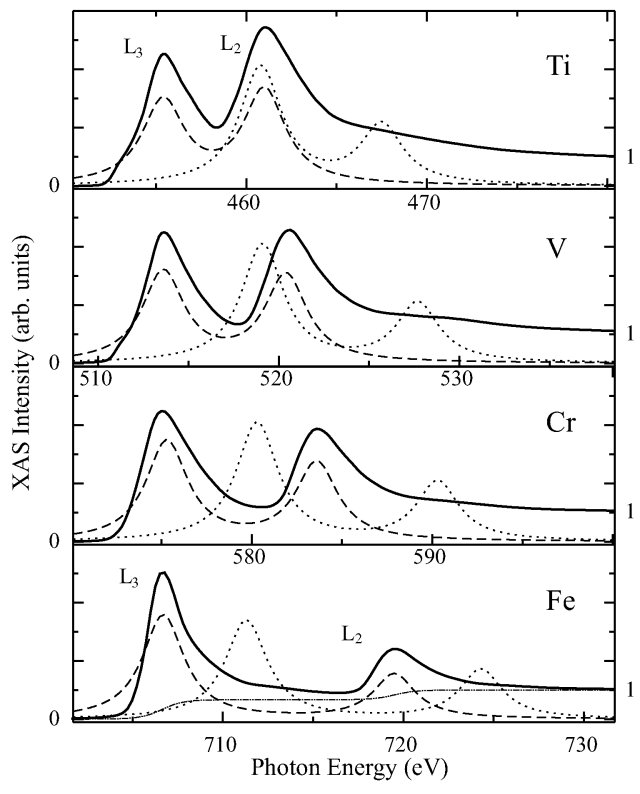

FIGURE 5. The experimental isotropic absorption spectra (solid line) at the $L_{2,3}$ edges are presented for the early $3 d$ $\mathrm{TMs} \mathrm{Ti}, \mathrm{V}$, and $\mathrm{Cr}$ versus Fe. The edge jumps are normalized to unity for direct comparison. The continuum in the experimental spectrum is simulated by a two-step function as shown for $\mathrm{Fe}$ (dashed-dotted line). The consideration of the core hole shifts the independent particle spectrum (dotted line) to lower photon energies and changes the branching ratio in the correlated spectrum (dashed line) as revealed by the DPA model [15].

$B_{\text {statistical }}=2 / 3$. When investigating the the isotropic data for the light $3 d$ metals given in Fig. 3 a strong deviation of the branching ratio from the statistical value is determined $(B(T i)=0.47, B(V)=0.51, B(C r)=0.56)$ [15]. This is due to the fact that spectral weight is shifted from the $L_{3}$ edge to the $L_{2}$ edge the lighter the $3 d$ element becomes. These effects are assigned to core-hole correlation effects. The spin-orbit splitting of the initial $2 p_{3 / 2}$ and $2 p_{1 / 2}$ states reduces toward the lighter $3 d$ elements which has the consequence that the two excitations couple. To model this effect we established a three-level or double-pole approximation (DPA) to the time-dependent density functional theory (TDDFT) as schematically presented in Fig. 4 [15]. Here, the x-ray absorption is dominated by two poles corresponding to the two absorption edges $L_{3}$ and $L_{2}$ which are strongly coupled. All other excitations are neglected in this approximation since they are weakly coupled to these two excitations.

A more detailed and quantitative investigation of the branching ratio is possible using the linear densitydensity response function $\chi$ to a small frequencydependent perturbation $[15,16,17]$. Within the framework of TDDFT $\chi$ is related to the response function $\chi_{s}$
TABLE 1. Excitation energies in $\mathrm{eV}$ obtained from $\mathrm{KS}$ calculations $\left(\omega_{i}^{K S}\right)$ and from experiment $\left(\Omega_{i}\right)$, experimental branching ratio $B$ and matrix elements $K_{i j}$ [15]. The experimental error of $\Omega_{i}$ is below $10^{-3}$, the one of $B$ in the order of $1 \%$.

\begin{tabular}{lcccccccc}
\hline $3 d \mathrm{TM}$ & $\omega_{1}^{K S}$ & $\omega_{2}^{K S}$ & $\Omega_{1}$ & $\Omega_{2}$ & $B$ & $K_{11}$ & $K_{22}$ & $K_{12}$ \\
\hline $22 \mathrm{Ti}$ & 460.8 & 467.5 & 455.4 & 461.0 & 0.47 & -2.57 & -3.34 & 0.54 \\
$23 \mathrm{~V}$ & 519.1 & 527.7 & 513.6 & 520.4 & 0.51 & -2.65 & -3.73 & 0.54 \\
$24 \mathrm{Cr}$ & 580.3 & 590.3 & 575.1 & 583.6 & 0.56 & -2.55 & -3.40 & 0.47 \\
$26 \mathrm{Fe}$ & 711.3 & 724.6 & 706.7 & 719.5 & 0.70 & -2.29 & -2.55 & -0.25 \\
\hline
\end{tabular}

of non-interacting particles via the Dyson-type equation

$$
\begin{aligned}
& \chi\left(\mathbf{r}, \mathbf{r}^{\prime}, \omega\right)=\chi_{s}\left(\mathbf{r}, \mathbf{r}^{\prime}, \omega\right) \\
& \quad+\int \mathrm{d}^{3} x \int \mathrm{d}^{3} x^{\prime} \chi_{s}(\mathbf{r}, \mathbf{x}, \omega) K\left(\mathbf{x}, \mathbf{x}^{\prime}, \omega\right) \chi\left(\mathbf{x}^{\prime}, \mathbf{r}^{\prime}, \omega\right)(1)
\end{aligned}
$$

The kernel that consists of the bare Coulomb interaction and the frequency-dependent XC kernel $f_{x c}\left(\mathbf{r}, \mathbf{r}^{\prime}, \omega\right)$ :

$$
K\left(\mathbf{r}, \mathbf{r}^{\prime}, \omega\right)=\frac{e^{2}}{\left|\mathbf{r}-\mathbf{r}^{\prime}\right|}+f_{x c}\left(\mathbf{r}, \mathbf{r}^{\prime}, \omega\right) .
$$

The exact exchange-correlation (XC) kernel describes the core-hole interaction with the photoelectron in addition to other many-body effects. If $K$ is neglected, the spectrum would reduce to the bare Kohn-Sham (KS) single particle spectrum represented by $\chi_{s}$. In XAS, the deviations produced by $K$ are called core-hole correlation effects. The basic idea of this DPA model is that we use the experimental values of the branching ratios and the level splittings and determine from those the matrix elements of the unknown XC kernel. The detailed DPA model is described in Ref. [15]. It turns out that the diagonal elements $K_{11}$ and $K_{22}$ are responsible for the shift of the KS levels to the correlated spectrum located at lower photon energies (see Fig. 5). The off-diagonal element $K_{12}$ leads to a shift of the spectral weight from the $L_{3}$ edge to the $L_{2}$ edge for the light $3 d$ elements and thereby changes the branching ratio. The values for the matrix elements determined by the procedure described above are given in table 1. Interestingly, we find that the value for $K_{12}$ is about a factor 5 smaller than the diagonal values and $K_{12}$ is basically constant for the light $3 d$ elements (about $0.5 \mathrm{eV}$ ). The DPA model reveals that the important measure for the coupling is $4\left|K_{12}\right| / \Delta \Omega$ (see Ref. [15]). Hence, it can be concluded that the reason for the increase of this property when traversing the $3 d$ series from $\mathrm{Fe}$ to $\mathrm{Ti}$ is only due to the decrease of the $2 p$ spin-orbit coupling. This demonstrates that the change of the branching ratio can be understood as "transition repulsion" when the two excitation energies get closer the lighter the $3 d$ elements are [15]. The XC kernel of TDDFT contains, in a very subtle and opaque way, all many-body effects beyond those of single-particle KS transitions. In general, even for weakly correlated systems, there will be small off-diagonal matrix elements 


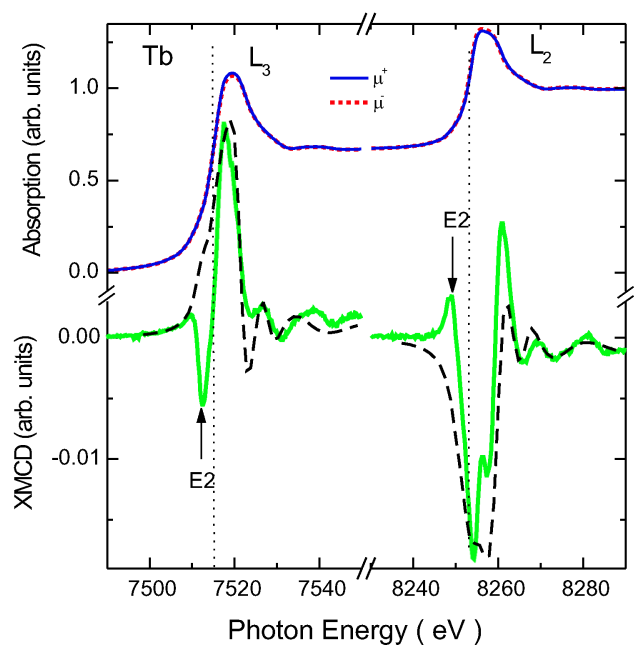

FIGURE 6. Normalized experimental Tb XAS (top) and XMCD spectra (bottom) at $10 \mathrm{~K}$ (solid lines), and theoretical XMCD spectra from FEFF8.20 (dashes). E2 labels $2 p \rightarrow 4 f$ quadrupolar transitions [20].

which can lead to the dramatic shifts in branching ratios shown here. The essential point of our DPA analysis is that this is not evidence of strong correlation. With this DPA model we achieved a transparent picture of the origin of the transfer of the spectral weight between the two edges and therefore of the change of the branching ratio for metals along the $3 d$ series. Furthermore, the determined matrix elements can be used to test future approximations of the exchange-correlation kernel.

\section{$5 d$ MAGNETISM IN RARE EARTH METALS}

It is clear that the magnetic properties of rare earth metals are determined by the $4 f$ levels. However, the magnetic ordering of these levels is mediated by the polarized $5 d$ band. To study the magnetism of the $5 d$ band the $L_{2,3}$ edges XMCD appears to be well suited. However, we have shown in the past that in addition to the electric dipolar contributions (E1: $2 p \rightarrow 5 d$ ) electric quadrupolar transitions (E2: $2 p \rightarrow 4 f$ ) can be identified in the rare earth $L_{2,3}$ edges XMCD [18, 7]. After separation of the two contributions we find that even the wrong sign of the $5 d$ moment is determined by means of the sum rules. This will be shown here for the $L_{2,3}$ edges XMCD of a Tb single crystal. In Fig. 6 the absorption coefficients for right and left circularly polarized light and the corresponding XMCD are depicted. The electric quadrupolar contributions were identified by calculations using the FEFF8 code [18, 19] and are marked by the arrows. This shows that the major contribution to the dichroic signal stems from the E1 transitions to the final $5 d$ states. Surprisingly, the main contribution to the XMCD at the $L_{3}$ edge is positive whereas a negative contribution is found at the $L_{2}$ edge. Applying the sum rule analysis this results in a $5 d$ moment which would be aligned antiparallel to the $4 f$ moments. However, this result is completely in contradiction to standard magnetometry results. The total moment of $\mathrm{Tb}$ is $9.34 \mu_{B}$ can be separated into a $4 f$ moment of $9.0 \mu_{B}$ within an atomic picture according to Hund's rules and a $5 d$ contribution of about $0.34 \mu_{B}$. Hence, a parallel alignment of the $4 f$ and $5 d$ moments is expected. How can one understand that the sum rule analysis even yields the wrong sign of the $5 d$ moment? In general the XMCD signal at the $L_{3}$ edge can be approximated by $\Delta \mu \approx\left[\mu^{\uparrow} \rho^{\uparrow}-\mu^{\downarrow} \rho^{\downarrow}\right] / 4$, where $\mu^{\uparrow}$ and $\mu^{\downarrow}$ are the (spin-dependent) dipole matrix elements and $\rho^{\uparrow}$ and $\rho^{\downarrow}$ describe the spin-dependent DOS. However, in the sum rule analysis it is assumed that the dichroic signal directly reflects the difference of the spin-dependent density of states $\Delta \mu \propto \rho^{\uparrow}-\rho^{\downarrow}$ by approximating $\mu^{\uparrow}=\mu^{\downarrow}$. Unfortunately, this assumption is not correct for the $L_{2,3}$ edges XMCD of rare earth elements. The $4 f-5 d$ interaction results in an attractive exchange potential for the spin-up $5 d$ electrons and a repulsive potential for the spin-down electrons. This has the consequence that $\mu^{\uparrow}>\mu^{\downarrow}$ [21]. To account for this difference we introduce a spin-asymmetry parameter $a_{j}[20,7]$ :

$$
a_{j} \equiv \frac{R_{j, n l \uparrow}-R_{j, n l \downarrow}}{R_{j, n l \uparrow}+R_{j, n l \downarrow}} .
$$

Here, $R_{j, n l \uparrow}$ is the matrix element for majority spin and $R_{j, n l \downarrow}$ that for the minority spin. The effect of the inequality of $\mu^{\uparrow}$ and $\mu^{\downarrow}$ is depicted in Fig. 7. The calculated spin-dependent DOS and the spin-asymmetry parameter using the FEFF8.2 code [19] are presented. The negative energies describe the occupied states and the positive energies characterize the unoccupied ones. We find that $a_{j}$ reaches a maximum of about 0.06 . Thereby the empty majority band is apparently enlarged as probed by the $\mathrm{XMCD}$ which results in a mostly positive XMCD contribution at the $L_{3}$ edge. Hence, the wrong sign of the $5 d$ moment is determined in the sum rule analysis. To overcome this difficulty we considered the spin-asymmetry parameter in the XMCD analysis. For that purpose we established a generalized form of the integral sum rules by adding spin-dependent correction terms (for details see [20]). The corrections to the spin sum rule are large when the number of $5 d$ holes $n_{h}(5 d)$ are large. The reason for the dramatic effect of the corrections (change of sign of $5 d$ moment) lies in the fact that for the $4 f$ rare metals like $\mathrm{Tb} n_{h}(5 d) \approx 9$. Applying these corrections we determine the following $5 d$ moments for the $\mathrm{Tb}$ spectra shown in Fig. 6: $\mu_{S}(5 d)=+0.37 \mu_{B}, \mu_{L}(5 d)=-0.027 \mu_{B}$ and therefore $\mu_{t o t}(5 d)=+0.34 \mu_{B}$. The positive sign for $\mu_{S}$ 


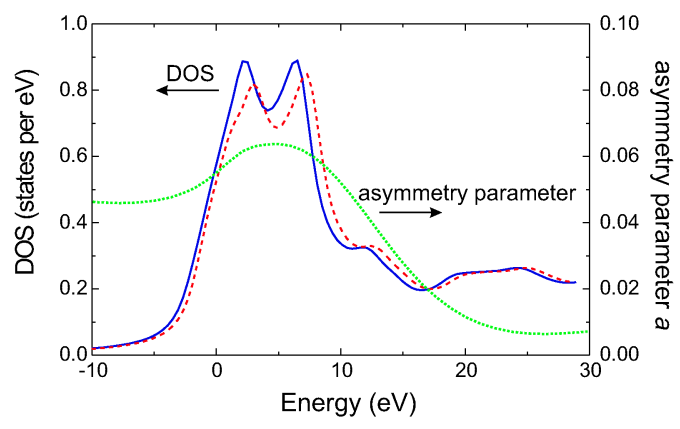

FIGURE 7. Density of states for spin-up (solid) and spindown (dashes) electrons in $\mathrm{Tb}$. The asymmetry parameter $a$ (dots) characterizes the difference from unity of the ratio between spin-up and -down matrix elements [Eq. (2)] and the zero of energy is the Fermi level $E_{F}$ [20].

and $\mu_{\text {tot }}$ shows that the $5 d$ moment is aligned parallel to the $4 f$ moments which is in good agreement with other magnetometries.

\section{CONCLUSION}

By examining two examples we have shown that the standard XMCD analysis can fail for magnetic systems of current interest. A systematic study along the $3 d$ series revealed that the sum rule analysis as well as the multipole-moment analysis breaks down for light $3 d$ elements. This failure could be related to the change of the branching ratio. To provide a transparent picture of the origin of this variation we developed a double-pole approximation within the TDDFT framework to describe the change of $L_{2,3} \mathrm{x}$-ray absorption spectra. Within this model we are able to determine the matrix elements of the unknown XC kernel. These values reveal that the shift of the spectral weight from the $L_{3}$ to the $L_{2}$ edge is primarily due to the reduced spin-orbit coupling. In the second example we focus on the $5 d$ magnetism of rare earth metals. We find by application of the integral sum rule analysis of the $L_{2,3}$ edges XMCD that even the wrong sign of the $5 d$ moment is determined. This is due to the spin-dependence of the transition matrix elements. The inclusion of this effect in the analysis yields the correct $5 d$ moments in agreement with other magnetometries. Both examples reveal that only by comparison of the experimental spectra to calculations of the $\mathrm{x}$-ray absorption coefficients and the corresponding dichroic spectra the magnetic ground state properties can be determined.

\section{ACKNOWLEDGMENTS}

The work was supported by BMBF (05 KS4 KEB/5) and DFG (Heisenberg-Programm). Discussions with P.M. Oppeneer and O. Eriksson are acknowledged.

\section{REFERENCES}

1. J. L. Erskine and E. A. Stern, Phys. Rev. B 12, 5016-5024 (1975).

2. G. Schütz, W. Wagner, W. Wilhelm and P. Kienle, Phys. Rev. Lett. 58, 737-740 (1987).

3. B. T. Thole, P. Carra, F. Sette and G. van der Laan, Phys. Rev. Lett. 68, 1943-1946 (1992).

4. P. Carra, B. T. Thole, M. Altarelli and X. Wang, Phys. Rev. Lett. 70, 694-697 (1993).

5. G. van der Laan, Phys. Rev B 55, 8086-8089 (1997).

6. H. Ebert, Rep. Prog. Phys. 59, 1665-1735 (1996).

7. H. Wende, Rep. Prog. Phys. 67, 2105-2181 (2004).

8. A. L. Ankudinov and J. J. Rehr, Phys. Rev. B 51, 1282-1285 (1995).

9. Y. Teramura , A. Tanaka and T. Jo, J. Phys. Soc. Japan 65, 1053-1055 (1996).

10. A. Scherz, P. Poulopoulos, H. Wende, G. Ceballos and K. Baberschke, J. Appl. Phys. 91, 8760-8762 (2002).

11. A. Scherz, H. Wende, K. Baberschke, J. Minár, D. Benea and H. Ebert, Phys. Rev. B 66, 184401-1-7(2002).

12. I. Mirebeau, G. Parette and J. W. Cable, J. Phys. F: Met. Phys. 17, 191 (1987).

13. A. Scherz, H. Wende and K. Baberschke, Appl. Phys. A 78, 843-846 (2004).

14. A. Scherz, H. Wende, C. Sorg, K. Baberschke, J. Minár, D. Benea and H. Ebert, Physica Scripta T115, 586-588 (2005).

15. A. Scherz, E. K. U. Gross, H. Appel, C. Sorg, K. Baberschke, H. Wende, and K. Burke Phys. Rev. Lett. 95, 253006-1-4 (2005).

16. J. Schwitalla and H. Ebert, Phys. Rev. Lett. 80, 4586-4589 (1998).

17. A.L. Ankudinov, A.I. Nesvizhskii, and J.J. Rehr, Phys. Rev. B 67, 115120-1-6 (2003).

18. H. Wende, Z. Li, A. Scherz, G. Ceballos, K. Baberschke, A. Ankudinov, J. J. Rehr, F. Wilhelm, A. Rogalev, D. L. Schlagel and T. A. Lograsso, J. Appl. Phys. 91, 7361-7363 (2002).

19. A. L. Ankudinov, B. Ravel, J. J. Rehr, and S. D. Conradson, Phys. Rev. B 58, 7565-7576 (1998).

20. A. L. Ankudinov, J. J. Rehr, H. Wende, A. Scherz and K. Baberschke, Europhys. Lett. 66, 441-447 (2004).

21. X. Wang, T. C. Leung, B. N. Harmon, and P. Carra, Phys. Rev. B 47, 9087-9090 (1993). 